\title{
CERTAIN FORMAL INVARIANCES IN BOOLEAN ALGEBRAS*
}

\author{
BY \\ NORBERT WIENER
}

Many mathematical systems are defined through postulates concerning (1) a class $(K)$ of elements $(a, b, c, \cdots)$, and (2) a certain definite group of relations or rules of combination joining these elements to one another.

These relations or rules of combination form only a part of those which may be said to belong to the system, for the latter may be considered to contain all those relations and rules which may be defined as logical functions of those with which the postulates deal and of certain selected elements of the system, and of nothing else. Thus, ordinary real algebra may be defined by postulates involving only the rules of combination + and $\times$, but it also contains as operations the rules of combination which, when applied to $x$ and $y$, yield us $x^{2}+2 x y$, or $x+1 / y$, or $x^{y}$. It consequently becomes an interesting question whether the postulates of a given system deal with relations or rules of combination whose position in the system is absolutely different from that of any of the other relations or rules belonging to the system; and if this is not so, it is natural to ask what other relations or rules of the system have formal properties similar to those of the entities which form the subject-matter of the postulates; for it would seem that a system whose postulates deal with entities which occupy a unique position in it has in some sense received a more thoroughgoing analysis than one where this is not the case. As the formal properties of the relations and operations in terms of which a system is defined, in so far as they are determinate, are given in the postulates, this question reduces itself to the investigation of what relations or operations of the system satisfy the postulates. We shall discuss this question in the case of the boolean algebras, although in a somewhat limited form.

This limitation consists in a somewhat narrower definition of the statement that an operation belongs to a given boolean algebra. We shall say that an operation belongs to a boolean algebra if it is the result of the performance of any finite sequence of the operations of the algebra-logical addition, logical multiplication, and negation-on the operands and certain specified "constant" elements of the algebra. That is, if it can be represented in the

\footnotetext{
* Presented to the Society, December 27, 1916.
} 
established symbolism of the algebra. It was pointed out by Boole* that if we represent the "logical sum" of $x$ and $y$ by $x+y$, their "logical product" by $x y$, and the negation of $x$ by $\bar{x}$, any operation on $x$ and $y$ may be written in the form $A x y+B x \bar{y}+C \bar{x} y+D \bar{x} \bar{y}$, where $A, B, C$, and $D$ are any elements of the algebra you choose. E. V. Huntington has shown $\dagger$ that a set of postulates may be developed for a boolean algebra in terms of the relation of logical addition alone. Our investigation will hence consist in seeing what conditions the coefficients $A, B, C$, and $D$ must fulfil in order that the operation $A x y+B x \bar{y}+C \bar{x} y+D \bar{x} \bar{y}$ may satisfy the conditions expressed in these postulates.

In the paper referred to above, Huntington says: ". . We take as the fundamental concepts a class, $K$, with a rule of combination, $\oplus$; and as the fundamental propositions, the following nine postulates:

" $A$. $a \oplus a=a$ whenever $a$ and $a \oplus a$ belong to the class.

" $B$. $a \oplus b=b \oplus a$ whenever $a, b, a \oplus b$, and $b \oplus a$ belong to the class.

“ $C .(a \oplus b) \oplus c=a \oplus(b \oplus c)$ whenever $a, b, c, a \oplus b, b \oplus c,(a \oplus b)$ $\oplus c$, and $a \oplus(b \oplus c)$ belong to the class.

" $D$. There is an element $\wedge$ such that $a \oplus \wedge=a$ for every element $a$.

" $E$. There is an element $\vee$ such that $\vee \oplus a=\vee$ for every element $a$.

" $F$. If $a$ and $b$ belong to the class, then $a \oplus b$ belongs to the class.

" $G$. If the elements $\wedge$ and $\vee$ in Postulates $D$ and $E$ exist and are unique, then for every element $a$ there is an element $\bar{a}$ such that $\left(1^{\circ}\right)$ if $x \oplus a=a$ and $x \oplus \bar{a}=\bar{a}$, then $x=\wedge$; and $\left(2^{\circ}\right) a \oplus \bar{a}=\vee$.

" $H$. If Postulates $A, D, E$, and $G$ hold, and if $a \oplus \bar{b} \neq \bar{b}$, then there is an element $x \neq \wedge$ such that $a \oplus x=a$ and $b \oplus x=b$.

" $J$. There are at least two elements, $x$ and $y$, such that $x \neq y$. ."

Huntington's $\oplus$ is meant to represent the operation of logical addition. We shall use $\oplus$ as a symbol for any operation in the algebra of logic which possesses such formal properties as to satisfy Huntington's postulates, and shall express logical addition and multiplication proper by the same symbols and according to the same conventions as those used in ordinary algebra. We shall indicate negation by a superposed bar, and the operation which takes the place of negation when $\oplus$ is substituted for + by an accent.

If we take $A x y+B x \bar{y}+C \bar{x} y+D \bar{x} \bar{y}$ as our $x \oplus y$, Huntington's postulate $A$ becomes:

$$
A a+D \bar{a}=a .
$$

As this is true independently of the value of $a$, we may substitute for it the universe, 1 , or the null-class, 0 . We thus see that $A=1$ and that $D=0$. The relation $x \oplus y$ can consequently be written in the form $x y+B x \bar{y}+C \bar{x} y$.

* Laws of Thought, pp. 73-78.

$\dagger$ These Transactions, vol. 5 (1904), pp. 306-308. 
Postulate $B$ tells us that our operation is symmetrical with regard to $x$ and $y$. Consequently $B=C$, and $x \oplus y$ reduces itself to the form

$$
x y+B(x+y) \text {. }
$$

Postulate $C$ is the law of associativity. $\quad a \oplus(b \oplus c)$ becomes

$$
a[b c+B(b+c)]+B[a+b c+B(b+c)] .
$$

This reduces to $a b c+B(a+b+c)$. As this is a symmetrical function of $a, b$, and $c$, it is equivalent to $c \oplus(a \oplus b)$, and this, by postulate $B$, is but another form of $(a \oplus b) \oplus c$. Postulate $C$ consequently imposes no restriction on an operation in the algebra of logic that is not already imposed by postulates $A$ and $B$.

Postulate $D$ is satisfied by all functions of the form $a b+B(a+b)$, for $a \bar{B}+B(a+\bar{B})=a \bar{B}+a B=a$. Thus $\bar{B}$ is a possible value of our $\wedge$. Furthermore, there is no other entity with this property. For let $x$ be such an entity. Then we may substitute 1 for $a$, and we get

$$
1=1 x+B(1+x)=x+B \text {. }
$$

Substituting 0 for $a$, then

$$
0=0 x+B(0+x)=B x .
$$

This proves that $\bar{B}$ is the only value which $x$ can assume.

Postulate $E$ is always satisfied by functions of the form $x y+B(x+y)$, for $a B+B(a+B)=B$. It is also true that, if $a x+B(a+x)=x$, whatever $a$ may be, then $x=B$. For let $a=1$. Then

If $a=0$,

$$
x=1 x+B(1+x)=x+B .
$$

$$
x=0 x+B(0+x)=B x .
$$

From the first equation,

$$
B x=B(x+B)=B .
$$

Putting these results together, we see that $x$ must equal $B$, which is therefore the only possible value of $\mathrm{V}$.

Postulate $F$ is satisfied by all functions in the algebra of logic, as one may see on inspection. This fact enables us to neglect the hypothesis which Huntington attributes to postulates $A, B$, and $C$, and thus proves that for $x \oplus y$ to satisfy Huntington's postulates not only is it sufficient that it be of the form $x y+B(x+y)$, but also necessary, as is shown by a brief consideration of the theorems we have already proved.

As the hypothesis of postulate $G$ has been shown to be satisfied by what we have already proved, we need only consider its conclusion. We then find 
that this postulate too is satisfied by all expressions of the form

$$
x y+B(x+y) .
$$

If we make our $\bar{a}$ of the $(+, \times)$ system the element which Huntington calls $\bar{a}$ and which we have agreed to call $a^{\prime}$ to avoid confusion, we discover that if $x \oplus a=a$ and $x \oplus \bar{a}=\bar{a}$, then

$$
a x+B(x+a)=a, \quad \text { and } \quad \bar{a} x+B(x+\bar{a})=\bar{a} .
$$

When we transform these into equations to ${ }_{0}$, they become, respectively,

$$
B x \bar{a}+\bar{B} \bar{x} a=0 \quad \text { and } \quad B x a+\bar{B} \bar{x} \bar{a}=0 .
$$

Putting these together, we see that $B x+\bar{B} \bar{x}=0$, so that $x=\bar{B}$, which we have found to be our $\wedge$. Furthermore,

$$
a \oplus \bar{a}=a \bar{a}+B(a+\bar{a})=0+B 1=B=\vee .
$$

Thus the negation of our original algebra may still remain the negation of any of our new boolean algebras, and all operations of the form $x y+B(x+y)$ satisfy postulates $A-G$, while no other operations in the algebra have this property. It is further true that the negation of our original algebra can be replaced by no other operation definable as an operation belonging to the algebra of logic. As is well known, all operations belonging to the algebra of logic, in the sense in which this phrase is usually taken, may be written in the form $M x+N \bar{x}$, if they are operations on the single variable $x$. Let our $a^{\prime}$ be expressed thus as $M a+N \bar{a}$. Postulate $G$ tells us $\left(1^{\circ}\right)$ that if

and

$$
x a+B(x+a)=a,
$$

$$
x(M a+N \bar{a})+B[x+(M a+N \bar{a})]=M a+N \bar{a},
$$

then $x$ must equal $\bar{B}$. The hypothesis may be rewritten in the combined form

or

$$
B x \bar{a}+\bar{B} \bar{x} a+B x(\bar{M} a+\bar{N} \bar{a})+\bar{B} \bar{x}(M a+N \bar{a})=0,
$$

$$
x(B \bar{a}+B \bar{M})+\bar{x}(\bar{B} a+\bar{B} N)=0 .
$$

Since $(B \bar{a}+B \bar{M})(\bar{B} a+\bar{B} N)=0$, this equation is always solvable for $x$. Solving it, we get

$$
x=\bar{B} a+\bar{B} N+u(\bar{B}+a M)=\bar{B}(a+N+u)+u a M,
$$

where $u$ is indeterminate. As $x$ must equal $\bar{B}$, we obtain the equation

$$
\bar{B}=\bar{B}(a+N+u)+u a M, \quad \text { or } \quad B u a M+\bar{B} \bar{u} \bar{a} \bar{N}=0 .
$$

As $a$ and $u$ are both indeterminate, we may assign them both the value 0 , whence we get $\bar{B} \bar{N}=0$, or the value 1 , whence $B M=0$. The second part 
of $G$ yields us the formula

$$
a(M a+N \bar{a})+B(a+M a+N \bar{a})=B, \quad \text { or } \quad M a \bar{B}+\bar{N} \bar{a} B=0 .
$$

As $a$ is indeterminate, we can make it either 1 or 0 , thus getting the results $M \bar{B}=0$ and $\bar{N} B=0$. Putting these results together with those which we have just reached from the first part of $G$, we see that $M=0, N=1$, and $a^{\prime}=0 a+1 \bar{a}=\bar{a}$. We thus see that the only operation in the algebra of logic which can have the characteristic properties of negation in a system whose operations are such that they can be expressed in the symbolism of the algebra of logic is the operation of negation itself.

Postulate $H$ imposes no further restriction on $\oplus$. It tells us that if

$$
a \bar{b}+B(a+\bar{b}) \neq \bar{b},
$$

there is some element $x$ other than $\bar{B}$, such that

$$
a x+B(a+x)=a, \quad \text { and } \quad b x+B(b+x)=b .
$$

Now $a b+\bar{B}(a+b)$ is such an $x$. In the first place, it cannot equal $\bar{B}$, for if it did, the equation $a b+\bar{B}(a+b)=\bar{B}$, which we should obtain, would reduce to the form $B a b+\bar{B} \bar{a} \bar{b}=0$, whence we should get the solution $a=\bar{B} \bar{b}+u(\bar{B}+\bar{b})$, where $u$ is indeterminate. Substituting this in the inequality $a \bar{b}+B(a+\bar{b}) \neq \bar{b}$, we get

$$
\bar{B} \bar{b}+B(\bar{B} \bar{b}+u(\bar{B}+\bar{b})+\bar{b}) \neq \bar{b} .
$$

This is reducible to the form $\bar{b} \neq \bar{B} \bar{b}+B \bar{b}$, which is manifestly false. Consequently $a b+\bar{B}(a+b)$ is a different element from $\bar{B}$. Furthermore, we have

$$
\begin{aligned}
a x+B(a+x)=a[a b+\ddot{B}(a+b)]+B[a+a b & +\bar{B}(a+b)] \\
& =a b+\bar{B} a+B a=a .
\end{aligned}
$$

Similarly, $b x+B(b+x)=b$. This proves our point.

Postulate $J$ is obviously satisfied by our operation $\oplus$, just because it is satisfied by our original boolean algebra. We have consequently proved that the necessary and sufficient condition that an operation expressible in the symbolism of a boolean algebra should possess the formal properties of the + operation is that it should be of the form $x y+B(x+y)$, and that a transformation of the ordinary addition into this new operation leaves the operation of negation unaltered. As a consequence the operation made to correspond to logical multiplication by this transformation will be that which, when applied to $x$ and $y$, yields us

$$
\overline{(\bar{x} \oplus \bar{y})}=[\bar{x} \bar{y}+B(\bar{x}+\bar{y})]=(x+y)(\bar{B}+x y)=x y+\bar{B}(x+y) .
$$


It is a well-known fact that all operations of the form $\bar{A} x+A \bar{x}$ are oneone, and that these are the only one-one operations in a boolean algebra.* It is obvious that if the whole of a boolean algebra is transformed into itself by one of these transformations, all its operations will be changed into relations of similar formal properties. The question thus arises, what relation will hold between $\bar{A} x+A \bar{x}, \bar{A} y+A \bar{y}$, and $\bar{A} z+A \bar{z}$ if $x+y=z$ ? Let $\bar{A} x+A \bar{x}=u, \bar{A} y+A \bar{y}=v$, and $\bar{A} z+A \bar{z}=w$. Then $x=\bar{A} u+A \bar{u}$, $y=\bar{A} v+A \bar{v}$, and $z=\bar{A} w+A \bar{w}$. But, by hypothesis, $x+y=z$. Therefore $\bar{A} u+A \bar{u}+\bar{A} v+A \bar{v}=\bar{A} w+A \bar{w}$. Hence

$$
\bar{A} u+\bar{A} v=\bar{A} w, \quad \text { and } \quad A \bar{u}+A \bar{v}=A \bar{w} .
$$

But $A w=A(A \bar{w})=A \overline{(A \bar{u}+A \bar{v})}=A(\bar{A}+u)(\bar{A}+v)=A u v$. Hence

$$
w=A w+\bar{A} w=A u v+\bar{A} u+\bar{A} v=u v+\bar{A}(u+v) .
$$

That is, the one-one operation which changes $x$ into $\bar{A} x+A \bar{x}$ transforms the operation of logical addition into the operation indicated by $u v+\bar{A}(u+v)$. Since the latter is in the general form for an operation which satisfies the postulates of a bcolean algebra and is expressible in the symbolism of a given boolean algebra, as we have already seen, we have shown that all such operations are "ordinaliy similar" $\dagger$ to the operation of logical addition. This we might also have deduced from the fact that an operation definable in the symbolism of a boolean algebra and satisfying Huntington's postulates is completely determinate when the element corresponding to 1 is given, so that there is only one operation which establishes this correspondence, namely, that which results from + when the whole algebra is subjected to a one-one transformation which makes the indicated change in 1 .

It is natural to consider those properties of the boolean algebras which are independent of the special $\oplus$-operation we take for our logical addition as more deep-rooted and fundamental than the rest, much as we assign some sort of a priority to those properties of a geometrical space which are unaltered by projection. It is to be noted that the invariant properties in the boolean algebras differ from those in geometry which are unaltered by projection in that the former are invariant with reference to all transformations expressed by formulas belonging to the algebra in question, so long as they leave its formal properties unchanged, while such an analytic-geometry transformation as $\left(x^{\prime}=x, y^{\prime}=y, z^{\prime}=z^{3}\right)$ does not alter the intrinsic formal properties of real space, though it alters the projective properties of certain configurations. It is a natural question to ask, whether a given relation between the entities of a boolean algebra retains its formal properties with respect to the algebra

* Schröder, Algebra der Logik, Vol. I, p. 463.

$\dagger$ Cf. Whitehead and Russell, Principia Mathematica, Vol. II, * 151. 
unaltered by all of the transformations definable in terms of the algebra which leave the formal character of the algebra unchanged, just as it is an important question in geometry whether a given relation remains unaltered by a projective transformation; but from what we have seen, the latter question is the more deep-rooted of the two. Now it is a well-known fact that every relation between entities belonging to a boolean algebra, which can be expressed at all in the symbolism of the algebra, can be expressed as a logical function of equations and inequations to 0 . We may therefore give a precise formulation to our question thus: when will an expression of the form

$$
\sum A_{a_{1}, a_{2}, \ldots, a_{k}} \prod_{l=1}^{l=k}\left(\bar{a}_{l} x_{l}+a_{l} \bar{x}_{l}\right)=0
$$

(where $k$ and $l$ are numerical subscripts and the summation sign extends over the $a$ 's which are either 1 or 0 ) retain its truth-value unaltered by any transformation of the $x$ 's which turns each $x_{k}$ into $\bar{B} x_{k}+B \bar{x}_{k}$, where $B$ is independent of the value of $k$ ? From what we have already proved, it may be seen that such expressions, and only logical functions of such expressions or their negations, represent relations among the $x$ 's which remain invariant with reference to all transformations definable in terms of the algebra, which leave it still a boolean algebra. If our expression is to remain invariant under all such transformations, it must retain its truth-value unaltered by the transformation of all the $x$ 's into their negations. This transforms our relation into

$$
\sum A_{a_{1}, a_{2}, \ldots, a_{k}} \prod_{l=1}^{l=k}\left(a_{l} x_{l}+\bar{a}_{l} \bar{x}_{l}\right)=0
$$

From this and our original equation we can derive an equivalent equation by adding the expressions equated to 0 . This equation will read

$$
\sum A_{a_{1}, a_{2}, \ldots, a_{k}}\left[\prod_{l=1}^{l=k}\left(\bar{a}_{l} x_{l}+a_{l} \bar{x}_{l}\right)+\prod_{l=1}^{l=k}\left(a_{l} x_{l}+\bar{a}_{l} \bar{x}_{l}\right)\right]=0 .
$$

This expression remains unchanged by all transformations of the $x$ 's which may be expressed in the symbolism of the algebra and still leave it a boolean algebra. As we have seen, any such transformation turns every $x$ into $\bar{B} x+B \bar{x}$, where $B$ is the same throughout the algebra. Also $\left(\bar{a}_{l} x_{l}+a_{l} \bar{x}_{l}\right)$ would be changed by this transformation into

$$
\bar{B}\left(\bar{a}_{l} x_{l}+a_{l} \bar{x}_{l}\right)+B\left(a_{l} x_{l}+\bar{a}_{l} \bar{x}_{l}\right),
$$

and $\left(a_{l} x_{l}+\bar{a}_{l} \bar{x}_{l}\right)$ would become

$$
B\left(\bar{a}_{l} x_{l}+a_{l} \bar{x}_{l}\right)+\bar{B}\left(a_{l} x_{l}+\bar{a}_{l} \bar{x}_{l}\right) .
$$


As a consequence,

becomes

$$
\prod_{l=1}^{l=k}\left(\bar{a}_{l} x_{l}+a_{l} \bar{x}_{l}\right)+\prod_{l=1}^{l=k}\left(a_{l} x_{l}+\bar{a}_{l} \bar{x}_{l}\right)
$$

$$
\begin{aligned}
\bar{B} \prod_{l=1}^{l=k}\left(\bar{a}_{l} x_{l}+a_{l} \bar{x}_{l}\right)+B \prod_{l=1}^{l=k}( & \left.a_{l} x_{l}+\bar{a}_{l} \bar{x}_{l}\right) \\
& +B \prod_{l=1}^{l=k}\left(\bar{a}_{l} x_{l}+a_{l} \bar{x}_{l}\right)+\bar{B} \prod_{l=1}^{l=k}\left(a_{l} x_{l}+\bar{a}_{l} \bar{x}_{l}\right),
\end{aligned}
$$

which is precisely its original value. This proves our point, and enables us to formulate the theorem that the necessany and sufficient condition that a relation between any number of the elements of a boolean algebra should remain invariant with reference to all transformations of the algebra into itself which may be expressed in its symbolism and leave it a boolean algebra is that the relation should remain invariant with regard to negation. Such a relation is fully characterized by the fact that it can be expressed as a logical function of equations or inequations to 0 of completely expanded functions of the related elements, in which the coefficient of each product of all the elements or their negations is identical with that of the product whose factors are the negations of those of the former one.

It will be noticed that Kempe's between-relation and obverse-relation* both satisfy this criterion, for Kempe's $a b \cdot c$, which is his way of saving that $c$ is between $b$ and $a$, may be written $a b \bar{c}+\bar{a} \bar{b} c=0$, while if $a, b, c$, etc., form an obverse collection, the sum of their product and that of their negations is 0 , and vice versa. It was, of course, recognized by Kempe that these relations, and all that can be defined in terms of them alone, represent a level of greater generality and universality in the boolean algebras than the operations of logical addition and logical multiplication, for the former do not enable us to discriminate between 0,1 , and the other elements of the system. What this article proves in addition is that Kempe's relations represent the very highest level of generality attainable in a boolean algebra, for they remain invariant under all the transformations of the algebra into another boolean algebra which may be expressed in the symbolism of the first algebra.

HARVARD UNIVERSITY, April 24, 1916

* A. B. Kempe, On a relation between the logical theory of classes and the geometrical theory of points, Proceedings of the London Mathematical Society, vol. 21 (1890), pp. 147-182. 